This item was submitted to Loughborough's Research Repository by the author.

Items in Figshare are protected by copyright, with all rights reserved, unless otherwise indicated.

\title{
Classification of instability modes in a model of aluminium reduction cells with a uniform magnetic field
}

PLEASE CITE THE PUBLISHED VERSION

http://dx.doi.org/10.1007/s00162-010-0201-y

\section{PUBLISHER}

(c) Springer-Verlag

VERSION

AM (Accepted Manuscript)

\section{PUBLISHER STATEMENT}

This work is made available according to the conditions of the Creative Commons Attribution-NonCommercialNoDerivatives 4.0 International (CC BY-NC-ND 4.0) licence. Full details of this licence are available at: https://creativecommons.org/licenses/by-nc-nd/4.0/

\section{LICENCE}

CC BY-NC-ND 4.0

\section{REPOSITORY RECORD}

Molokov, Sergei, G.A. El, and Alexander Lukyanov. 2019. "Classification of Instability Modes in a Model of Aluminium Reduction Cells with a Uniform Magnetic Field”. figshare. https://hdl.handle.net/2134/16940. 


\title{
Classification of instability modes in a model of aluminium reduction cells with a uniform magnetic field
}

\author{
Sergei Molokov ${ }^{1}$ Gennady El $^{2}$ Alexander Lukyanov $^{3}$ \\ ${ }^{1}$ Applied Mathematics Research Centre, Coventry University, UK \\ 2 Department of Mathematical Sciences, Loughborough University, UK \\ 3 Department of Mathematics, University of Reading, UK
}

\begin{abstract}
A unified view on the interfacial instability in a model of aluminium reduction cells in the presence of a uniform, vertical, background magnetic field is presented. The classification of instability modes is based on the asymptotic theory for high values of parameter $\beta$, which characterises the ratio of the Lorentz force based on the disturbance current, and gravity. It is shown that the spectrum of the travelling waves consists of two parts independent of the horizontal cross-section of the cell: highly unstable wall modes and stable or weakly unstable centre, or Sele's modes. The wall modes with the disturbance of the interface being localized at the sidewalls of the cell dominate the dynamics of instability. The Sele's modes are characterised by a distributed disturbance over the whole horizontal extent of the cell. As $\beta$ increases these modes are stabilized by the field.
\end{abstract}




\section{Introduction}

Aluminium is produced by decomposing alumina dissolved in a molten cryolite (sodium aluminium fluoride) by an electric current $I_{0}^{*}$ of $350-500 \mathrm{kA}$, which passes vertically down from the anode to the cathode (Fig. 1). As a result of this process, a two-layer fluid system is formed with molten aluminium at the bottom of the cell and a slightly lighter cryolite at the top. Aluminium is then siphoned from the cell periodically.

As cryolite is a very poor conductor, much of the energy supplied is wastefully released in the cryolite layer in the form of Joule heating. The desire to reduce the thickness of the cryolite layer, and thus to reduce the energy losses to a minimum, is limited by interfacial instabilities, which are magnetohydrodynamic (MHD) in nature. They result from the interaction of the disturbance current with the external magnetic field, which is induced in the external circuit supplying current to the cell [1]. This interaction creates the Lorentz force, which acts on aluminium. The resulting interfacial wave may amplify with time and lead to the instability. Therefore, it is vitally important to understand the nature of instabilities, and to find the ways to control them.

It is not surprising then that the interfacial instability of a two-layer system in the presence of a vertical current and a background magnetic field, has been studied by many authors, see a review in [2]. Various aspects of the problem, as well as possible sources of instability have been investigated. These are spatial noniniformities of the external magnetic field, various components of the field (horizontal/vertical), standing or travelling waves in bounded or unbounded domains, etc. Several methods to control the instability by mechanical means [3] and by a low-frequency alternating magnetic field [4] have also been proposed. 


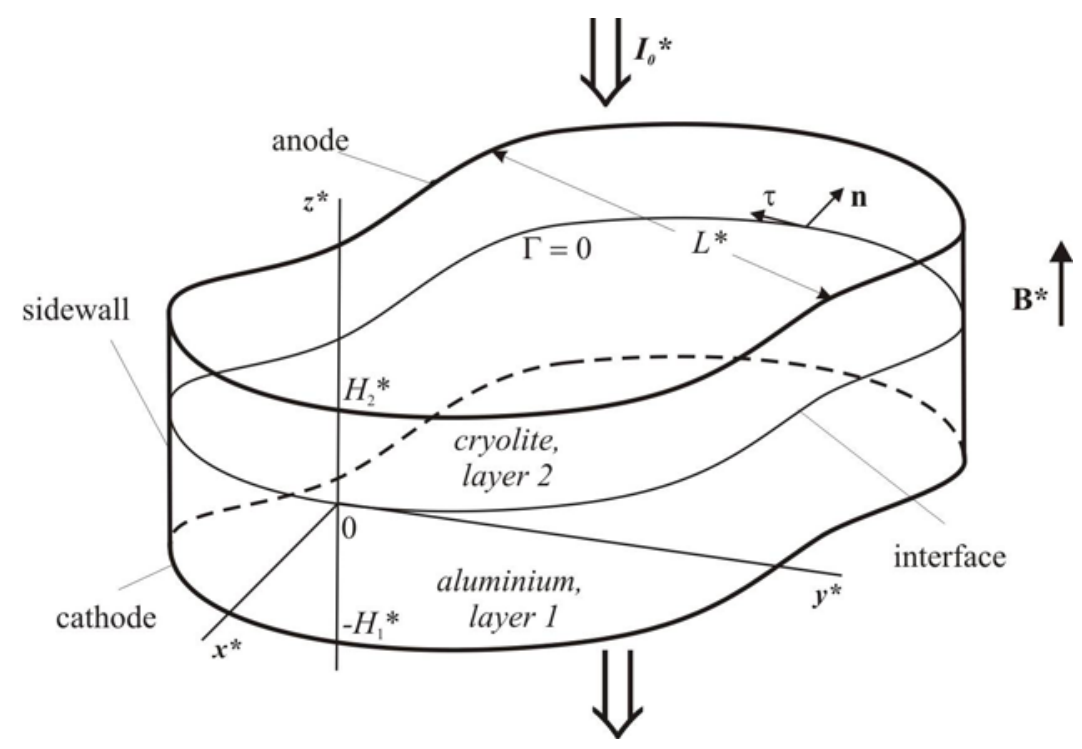

FIG. 1. Simplified schematic diagram of the aluminium reduction cell with horizontal crosssection given by an arbitrary function $\Gamma\left(x^{*}, y^{*}\right)=0$

A particular, widely used, simplified model of the MHD interaction within the cells leading to the instability has been suggested in [1], [5]-[7] and later employed in [8]-[16]. The model is based on the assumptions that the external, vertical magnetic field is uniform, and that the cell is completely covered by the anode. Concerning the nature of the instability within the limits of this model, main conclusions made by previous authors may be summarised as follows.

First of all, the external magnetic fields are highly complex, spatially varying within the cell. It has been recognized, however, that the most dangerous component of the field is vertical. It destabilizes the cell even if it is uniform [6].

Secondly, for certain cell geometries there is a critical value of the dimensionless parameter

$$
\beta=j_{0}^{*} B_{0}^{*} L^{* 2} /\left[H_{1}^{*} H_{2}^{*}\left(\rho_{1}^{*}-\rho_{2}^{*}\right) g^{*}\right]
$$

above which the disturbance of the interface starts growing in magnitude. In the above $j_{0}^{*}$ is the density of the supplied current, $B_{0}^{*}$ is the induction of the vertical component of the 
magnetic field, $L^{*}$ is a typical horizontal dimension of the cell, $H_{1}^{*}, \rho_{1}^{*}$ and $H_{2}^{*}, \rho_{2}^{*}$ are thicknesses and densities of aluminium and cryolite layers, respectively, and $g^{*}$ is gravitational acceleration. From the expression for $\beta$ it is clear that the reduction of the thickness of the cryolite layer, the increase of the current density, or the increase of the intensity of the background field has the same effect. Each of these actions leads to the increase in the parameter $\beta$, and may push its value above critical.

Parameter $\beta$ characterises the ratio of the Lorentz force based on the scalings of the disturbance current, $\eta_{0}^{*} j_{0}^{*} L^{* 2} /\left(H_{1}^{*} H_{2}^{*}\right)$, and vertical component of the magnetic field, $B_{0}^{*}$, on the one hand, and gravity, $\eta_{0}^{*}\left(\rho_{1}^{*}-\rho_{2}^{*}\right) g^{*}$, on the other. Here $\eta_{0}^{*}$ is the characteristic amplitude of the elevation of the interface. For $\beta>>1$ the electromagnetic effects dominate gravity. In aluminium reduction cells parameter $\beta$ varies in the range $6 \leq \beta \leq 340$ [14], [15], [2].

Finally, the equilibrium is most unstable to long waves with the wavelength comparable to the horizontal dimension of the cell.

It is important to note that the discussion above, as well as this investigation, is related to the MHD instability, which is always very long-wave and violent, as confirmed by a recent experiment [17]. The so-called MHD noise [18] involving waves of $0.5-2 \mathrm{~m}$ in length and of finite but small amplitude is always present during the smelting process, but it does not affect the stability of the cells and thus is of no concern here.

Several scenarios of the MHD instability have been proposed. Sneyd and Wang [6] and Bojarevics and Romerio [7] have independently proposed a model, later explored by Davidson and Lindsay [10], whereby the instability occurs owing to the magnetic coupling of gravity waves inside the whole domain occupied by the fluids. The interface becomes unstable owing to an internal resonance of these waves. 
This mechanism, however, has been questioned by Lukyanov, El and Molokov [12], who noted that the key coupling between gravity and the Lorentz force occurs at the sidewalls rather than inside the domain. It has been suggested that the instability in closed domains takes place as a result of multiple reflections and amplification of the waves at the sidewalls.

Further, an exact solution for a circle has been analysed in [12] for $\beta \gg>1$ for the modes corresponding to the most unstable, long waves. It has been shown that for these modes both the growth rate of instability and the frequency increase as $\sqrt{\beta / 2}$ independent of the mode number in the azimuthal direction, which gives a hint at universality of this dependence.

This universality has been confirmed independently by Morris and Davidson [13] using either $g^{*} \rightarrow 0$ of long-wave limits and by Molokov, El and Lukyanov [14] using the $\beta$ $>>1$ asymptotics, who have shown that the growth rate of the most unstable modes is proportional to $\sqrt{\beta / 2}$ independently of the geometry of the cell.

Thus there exist three different explanations of the instability. Here we reconcile the results obtained by previous authors and present a unified theory for this simplified model of the process based on the asymptotic analysis for high values of $\beta$. We show that all the modes of instability may be classified into two distinct groups independent of the cell geometry, and discuss mechanisms of instability for each group.

\section{Formulation}

It is assumed here that the vertical magnetic field $\mathbf{B}^{*}=B_{0}^{*} \mathbf{e}_{z}$ is uniform, and that the two-fluid system is contained in a finite or semi-infinite domain as shown in Fig. 1, where $\left(x^{*}\right.$, $\left.y^{*}, z^{*}\right)$ are Cartesian co-ordinates. The cell is supposed to be completely covered by the anode. Molten aluminium and cryolite form layers 1 and 2, respectively. Aluminium, cryolite, and 
cathode are characterised by the electrical conductivities $\sigma_{1}^{*}, \sigma_{2}^{*}$, and $\sigma_{c}^{*}$, respectively, which are ranked as follows: $\sigma_{2}^{*}<<\sigma_{c}^{*}<<\sigma_{1}^{*}$. The sidewalls of the box are electrically insulating. Typical values of parameters of the cell are: $H_{1}^{*}=0.3 m, H_{2}^{*}=0.05 m$, $\rho_{1}^{*}=2300 \mathrm{~kg} / \mathrm{m}^{3}, \quad \rho_{2}^{*}=2100 \mathrm{~kg} / \mathrm{m}^{3}, \quad \sigma_{1}^{*}=3.3 \cdot 10^{6}(\Omega \cdot \mathrm{m})^{-1}, \quad \sigma_{2}^{*}=200(\Omega \cdot \mathrm{m})^{-1}$, $\sigma_{c}^{*}=2 \cdot 10^{4}(\Omega \cdot m)^{-1}, L^{*}=10 m, B_{0}^{*}=3 \cdot 10^{-3}-10^{-2} T$, and $I_{0}^{*}=350-500 k A$.

We will be concerned with the stability of a plane interface located at $z^{*}=0$ in the long-wave approximation. The shallow-water model we use, based on the assumption $\varepsilon=H_{1}^{*} / L^{*}<<1$ (typically $\varepsilon \propto 0.03$ ), is essentially that developed by Urata [5] and is not derived here. For infinitesimally small perturbations of the interface the dimensionless, twodimensional, time dependent equations reduce to [12], [16]:

$$
\frac{\partial^{2} \eta}{\partial t^{2}}+\gamma \frac{\partial \eta}{\partial t}=\nabla^{2} \eta, \quad \nabla^{2} \phi=-\eta
$$

where time $t$, the elevation of the interface $\eta(x, y, t)$, and the electric potential in aluminium $\phi(x, y, t)$ are scaled with $L^{*} / c^{*}, \eta_{0}^{*}$, and $\eta_{0}^{*} j_{0}^{*} L^{* 2} /\left(\sigma_{1}^{*} H_{1}^{*} H_{2}^{*}\right)$, respectively. In the above $\gamma=\bar{\gamma}^{*} L^{*} / c^{*} \bar{\rho}^{*}$ is the dimensionless dissipation coefficient, $c^{*}=\left(g^{*} \Delta \rho^{*} / \bar{\rho}^{*}\right)^{1 / 2} \approx 20 \mathrm{~cm} / \mathrm{s}$ is the phase speed of the interfacial gravity waves, $\Delta \rho^{*}=\rho_{1}^{*}-\rho_{2}^{*}, \bar{\rho}^{*}=\rho_{1}^{*} / H_{1}^{*}+\rho_{2}^{*} / H_{2}^{*}$, $\bar{\gamma}^{*}=\rho_{1}^{*} \gamma_{1}^{*} / H_{1}^{*}+\rho_{2}^{*} \gamma_{2}^{*} / H_{2}^{*}$, and $\nabla \equiv \partial / \partial x \mathbf{e}_{x}+\partial / \partial y \mathbf{e}_{y}$. Assuming $\gamma_{1}^{*}=\gamma_{2}^{*}=0.03 s^{-1}$ as in [11], [14], gives $\gamma=\gamma_{1}^{*} L^{*} / c^{*} \approx 0.755$ as a typical value of the dimensionless dissipation coefficient $\gamma$

The boundary condition for the electrically insulating sidewalls and the nonpenetration condition at these walls yield

$$
\frac{\partial \phi}{\partial n}=0, \quad \frac{\partial \eta}{\partial n}=-\beta \frac{\partial \phi}{\partial \tau} \quad \text { at } \Gamma,
$$


respectively. Here function $\Gamma(x, y)=0$ defines the shape of the boundary (horizontal geometry of the cell); $\partial / \partial n$ and $\partial / \partial \tau$ are normal and tangential derivatives to $\Gamma=0$, respectively.

Eqs. (1) and (2) are the wave equation with linear damping and the Poisson equation for the electric potential in aluminium. Damping leads to the reduction of the growth rate and to a certain change in frequency of the waves. As this is not a crucial issue for this study, hereinafter we will assume that $\gamma=0$.

The electrical and mechanical parts of the problem are coupled in Eq. (1b) and in the boundary condition (2b). As we will see below, each type of coupling is associated with a particular group of instability modes. For both of them, however, coupling at the boundary is important. Indeed, in the absence of the background field, when $\beta=0$, the coupling at the boundary disappears, while that in Eq. (1b) remains. This gives pure gravity waves, satisfying the boundary condition

$$
\partial \eta / \partial n=0 \text { at } \Gamma
$$

The electric potential redistributes passively, following the differences in the elevation of the interface. Thus for $\beta \neq 0$ coupling at the boundary is essential. It vanishes if the boundary is removed to infinity, which explains why the equilibrium in an unbounded domain is stable [19].

Moreover, suppose for an instant that the sidewalls are perfect electric conductors. Then the boundary condition (2a) is replaced by

$$
\phi=0 \quad \text { along } \Gamma,
$$

while condition (2b) is reduced to Eq. (3). The problem for $\eta$ again decouples from that for $\phi$, implying no instability. It will be shown below that this is what happens for a particular group of instability modes in the limit $\beta \rightarrow \infty$. 
To summarize, for instability to occur it is essential not only that the boundary is present, but that it is electrically insulating [13], [14]. These observations are crucial for further understanding of the nature of the unstable modes, as well as for the explanation of the fact that some modes develop strong growth, while the other ones are either weakly unstable or stable. This will be shown for several geometries of the cell.

In what follows we analyse first the exact solutions for a half-plane and for a circle for $\beta>>1$, and then develop an asymptotic solution for an infinite channel drawing attention to the common features of the instability modes for these geometries. Finally we will discuss the results obtained both here and previously and will develop a unified view of the mechanisms of instability for each group of modes.

\section{Half-plane}

The most basic geometry with a sidewall present is a half-plane $x<0$, where the twofluid layer is bounded by a sidewall at $x=0$ (Fig. 2). For this geometry the boundary conditions (2) are:

$$
\frac{\partial \phi}{\partial x}=0, \quad \frac{\partial \eta}{\partial x}=-\beta \frac{\partial \phi}{\partial y} \quad \text { at } \quad x=0
$$

We are looking for a solution in the form of travelling waves

$$
\eta=\hat{\eta}(x) \exp \left(i k_{y} y-i \omega t\right), \quad \phi=\hat{\phi}(x) \exp \left(i k_{y} y-i \omega t\right),
$$

where $k_{y}>0$ is a real wavenumber, and $\omega$ is a complex frequency. 


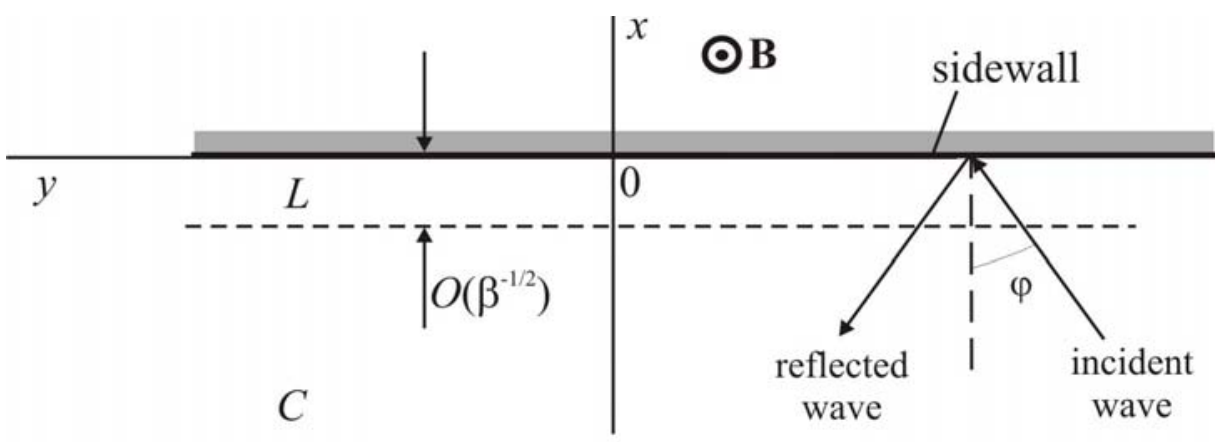

fluids

FIG. 2. Schematic diagram of the half-plane problem. On the left are flow subregions for the wall modes for $\beta>>1$ : boundary layer $L$ and core $C$; on the right is schematic diagram of reflection of a plane wave from the sidewall.

Substituting Eqs. (6a,b) into Eqs. (1) and boundary conditions (5a,b) yields:

$$
\begin{aligned}
& \frac{d^{2} \hat{\eta}}{d x^{2}}+k_{x}^{2} \hat{\eta}=0, \quad \frac{d^{2} \hat{\phi}}{d x^{2}}-k_{y}^{2} \hat{\phi}=-\hat{\eta}, \\
& \frac{d \hat{\phi}}{d x}=0, \quad \frac{d \hat{\eta}}{d x}=-i \beta k_{y} \hat{\phi} \quad \text { at } \quad x=0,
\end{aligned}
$$

where

$$
k_{x}^{2}=\omega^{2}-k_{y}^{2} .
$$

The solution is normalized in such a way that

$$
\hat{\eta}(0)=1
$$

As the domain is semi-infinite, the spectrum of the problem consists of a continuous and a discrete part.

\subsection{Continuous spectrum: reflection of a plane wave from a sidewall}

Assuming that both $\omega$ and $k_{x}$ are real yields the solution of Eqs. (7)-(10) as follows:

$$
\hat{\eta}=C_{1} e^{i k_{x} x}+C_{2} e^{-i k_{x} x},
$$




$$
\hat{\phi}=-i \frac{k_{x}}{\omega^{2} k_{y}}\left(C_{1}-C_{2}\right) e^{k_{y} x}+\frac{1}{\omega^{2}}\left\{C_{1} e^{i k_{x} x}+C_{2} e^{-i k_{x} x}\right\}
$$

where $\quad C_{1,2}=\frac{1}{2}\left\{1 \mp \frac{\beta k_{y}}{k_{x}\left(\omega^{2}-\beta i\right)}\right\}$. One of the exponential terms in Eq. (11) is the incident wave on the sidewall with the angle of incidence $-\pi / 2<\varphi<\pi / 2$ given by the expression $\tan \varphi=k_{y} / k_{x}$, while the other one is a reflected wave.

The reflection coefficient $\mu$ is defined as follows:

$$
\mu=\frac{\left|C_{2}\right|^{2}}{\left|C_{1}\right|^{2}}=\frac{\beta_{\lambda}^{2}+\left(1+\beta_{\lambda} \tan \varphi\right)^{2}}{\beta_{\lambda}^{2}+\left(1-\beta_{\lambda} \tan \varphi\right)^{2}}
$$

where $\beta_{\lambda}=\beta /\left(k_{x}^{2}+k_{y}^{2}\right)=\beta \sin ^{2} \varphi / k_{y}^{2}$ is the value of parameter $\beta$ defined with the wavelength in the direction of propagation of the incident wave scaled with $2 \pi$.

From Eq. (13) follows that $\mu>1$ for $0<\varphi<\pi / 2$, which corresponds to the amplification of the wave propagating in the $+y$-direction. Reversely, if $-\pi / 2<\varphi<0$, then $\mu<1$ corresponding to suppression of the wave propagating in the $-y$-direction.

Variation of $\mu$ with $\varphi$ for several values of $\beta_{\lambda}$, and with $\beta_{\lambda}$ for several values of $\varphi$, are shown in Figs. 3 and 4, respectively. For a fixed value of $\beta_{\lambda}$ the reflection coefficient reaches a maximum of

$$
\mu_{\max }=\frac{1+\sqrt{1+\beta_{\lambda}^{2}}}{-1+\sqrt{1+\beta_{\lambda}^{2}}}
$$

at

$$
\varphi_{\max }=\arccos \frac{\beta_{\lambda}}{\sqrt{1+2 \beta_{\lambda}^{2}}}
$$




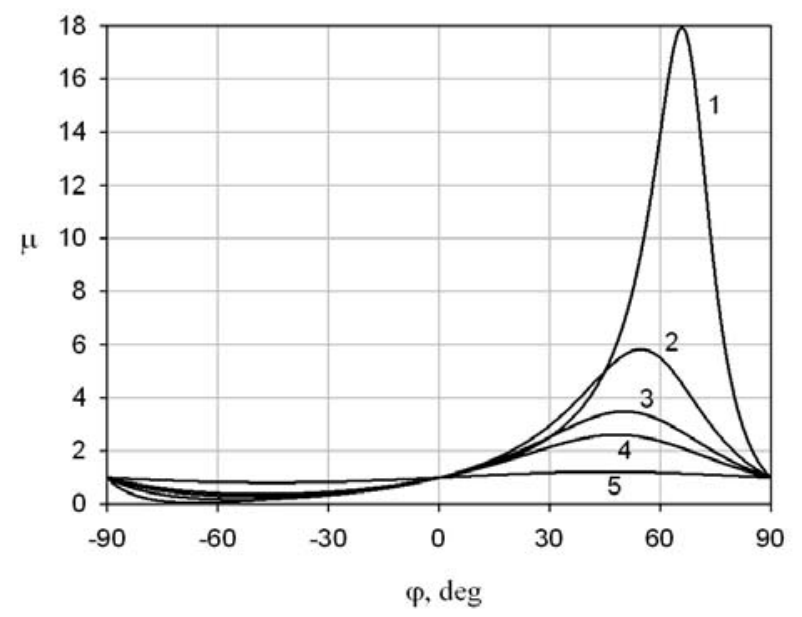

FIG. 3. Reflection coefficient as a function of $\varphi$ for several values of $\beta_{\lambda}: 0.5$ (1), 1 (2), 1.5 (3), 2 (4), 10 (5).

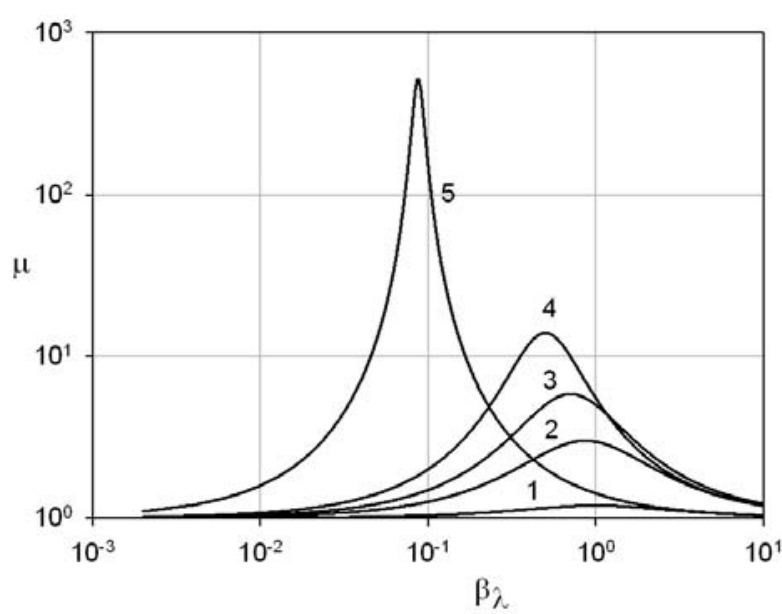

FIG. 4. Reflection coefficient as a function of $\beta_{\lambda}$ for several values of the reflection angle: $\varphi=5^{\circ}$ $(1), 30^{\circ}(2), 45^{\circ}(3), 60^{\circ}(4), 85^{\circ}(5)$.

The reflection angle for which the maximum is reached varies in the range $\pi / 4<\varphi_{\max }<\pi / 2$ for $\infty<\beta_{\lambda}<0$. The reflection coefficient increases indefinitely as $\beta_{\lambda} \rightarrow 0, \varphi_{\max } \rightarrow \pi / 2$, which implies that the short waves almost aligned with the sidewall are amplified most.

On the other hand,

$$
\mu_{\max } \cong 1+2 \beta_{\lambda}^{-1}+\ldots, \quad \varphi_{\max } \cong \pi / 4+\ldots \text { as } \beta_{\lambda} \rightarrow \infty,{ }^{\mathrm{i}}
$$



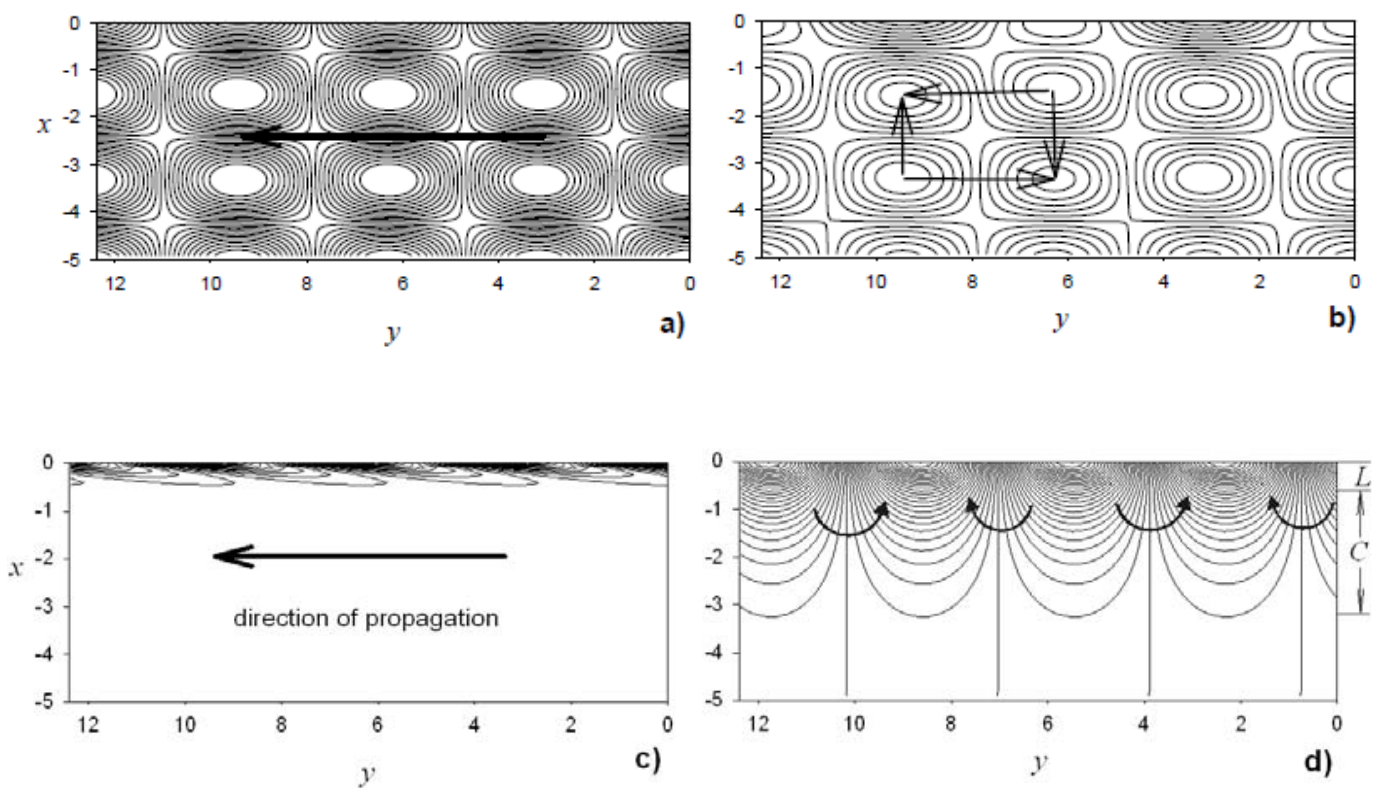

FIG. 5. Half-plane. Isolines of $\eta$ and $\phi$ for the Sele mode a) and b) and for the wall mode c) and d), respectively. Here $k_{y}=1, \beta=100, t=0$. Arrows in (b) and (d) show the direction of horizontal current.

implying that the amplification of the waves reduces to zero as the magnetic field induction increases.

For $\beta>>1$ and for $\tan \varphi=O(1)$ the leading terms in Eqs. (11) and (12) tend to become real functions. Then

$$
\begin{gathered}
\eta \cong \frac{\cos \left(k_{x} x-\varphi\right)}{\cos \varphi} \cos \left(k_{y} y-\omega t\right), \\
\phi \cong \frac{1}{\omega^{2}}\left\{-e^{k_{y} x}+\frac{\cos \left(k_{x} x-\varphi\right)}{\cos \varphi}\right\} \cos \left(k_{y} y-\omega t\right) .
\end{gathered}
$$

It is evident that both functions $\eta$ and $\phi$ are $O(1)$, and that their order of magnitude is thus determined by Eq. (1b), rather than the boundary condition (2b). Expressions (17) and (18) represent travelling waves in the $+y$-direction and standing waves in the $-x$-direction with functions of $x$ being their amplitudes. Thus for $\beta>>1$ the pattern for both $\eta$ and $\phi$ consists of channels along the $y$-axis, i.e. to waveguides, similar to the standard diffraction problem. This is shown in Fig. 5a,b for $\beta=100, \varphi=30^{\circ}$. The electric current in aluminium flows between 
crests and troughs of the wave in the whole domain as shown schematically in Fig. $5 \mathrm{~b}$. Sufficiently far from the wall the channels for $\phi$ coincide with those for $\eta$, and the solution tends to become that for the unbounded domain.

The exact expression for $\hat{\phi}(0)$ is:

$$
\hat{\phi}(0)=\frac{1}{\omega^{2}-\beta i},
$$

i.e. $\hat{\phi}(0) \cong i \beta^{-1} \rightarrow 0$ as $\beta \rightarrow \infty$. Thus, in the limit not only the normal, but also the tangential component of current, $\hat{j}_{y}=-i k_{y} \hat{\phi}$ vanishes at the boundary. As we will see later this is characteristic for what we will call the Sele modes of instability for closed domains.

\subsection{Discrete spectrum: wall modes}

Suppose now that both $k_{x}$ and $\omega$ are complex. Then the exponential functions in Eq. (11) describe either a spatially decaying, or growing disturbance away from the wall. Discarding the latter we set $C_{2}=0$ and require that $\operatorname{Im}\left(k_{x}\right)<0$.

Substituting Eq. (11) into Eq. (10) and using Eq. (12) yields:

$$
\hat{\eta}=\exp \left(i k_{x} x\right), \quad \hat{\phi}=\frac{1}{k_{x}^{2}+k_{y}^{2}}\left[\exp \left(i k_{x} x\right)-\frac{i k_{x}}{k_{y}} \exp \left(k_{y} x\right)\right]
$$

The boundary condition ( $8 \mathrm{~b}$ ) leads to a simple dispersion relation as follows:

$$
k_{x}\left(i k_{x}+k_{y}\right)=-\beta
$$

which gives

$$
k_{x} / k_{y}=\chi+i v
$$


where $\chi=-2^{-3 / 2} \sqrt{-1+\sqrt{16 \beta_{\lambda}^{2}+1}}, \quad v=\left(1-2^{-1 / 2} \sqrt{1+\sqrt{16 \beta_{\lambda}^{2}+1}}\right) / 2$. Similar to Sec. 3.1, parameter $\beta_{\lambda}=\beta / k_{y}^{2}$ is defined with the wavelength in the direction of propagation scaled with $2 \pi$.

Eqs. (9) and (22) yield two possible values for $\omega$, namely:

$$
\omega / k_{y}=\Omega_{r}+i \Omega_{i}
$$

where $\Omega_{r}= \pm 2^{-1 / 2} \sqrt{A+\sqrt{A^{2}+4 \chi^{2} v^{2}}}, \Omega_{i}=\chi v / \Omega_{r}$, and $A=\chi^{2}-v^{2}+1$.

The solution with positive and negative signs of $\Omega_{r}$ represents waves propagating in the $+y$ and $-y$-directions, respectively. The growth rate of the disturbance is determined by the imaginary part of $\omega$. For any non-zero value of $\beta$ the disturbance propagating in the $-y$ direction $\left(\Omega_{r}<0, \Omega_{i}<0\right)$ decays, while that in the $+y$-direction $\left(\Omega_{r}>0, \Omega_{i}>0\right)$ grows. Thus for any non-zero value of $\beta$ the equilibrium is unstable to travelling waves propagating in the $+y-$ direction.

The unstable wave is shown in Fig. $5 \mathrm{c}, \mathrm{d}$ for $\beta=100$ and for $k_{y}=1$. The contour lines of the disturbance of the interface exhibit characteristic tails, which will be observed in all the other flow geometries considered in this paper.

If MHD interaction is strong, i.e. $\beta_{\lambda}>>1$, two-term asymptotic expansions of $k_{x}$ and $\omega$ are as follows:

$$
k_{x}=-\sqrt{\beta i}+\frac{1}{2} k_{y} i+O\left(\beta^{-1 / 2}\right), \quad \omega= \pm\left[\sqrt{\beta i}-\frac{1}{2} k_{y} i+O\left(\beta^{-1 / 2}\right)\right] .
$$

Throughout this paper $\sqrt{ \pm i}=(1 \pm i) / 2$, i.e. the branches of the roots with positive real part are taken. For consistency with the shallow-water approximation we require that $\sqrt{2 / \beta}>>\varepsilon$, 
which formally places an upper limit on the value of $\beta$. For $\varepsilon=0.03$ this gives $\beta<<2000$, i.e. the theory presented here is valid for all practical purposes.

Calculations by Kohno and Molokov [16] show that the asymptotic expressions (24) with two terms and the wavenumber corresponding to the perimeter of the domain give accurate results for $\beta>6$ for any closed geometry of the cell.

Substituting one-term asymptotics for $k_{x}$ from Eq. (24a) into Eqs. (20), gives expressions for $\hat{\eta}$ and $\hat{\phi}$ as follows:

$$
\hat{\eta} \cong \hat{\eta}_{L}=\exp \lfloor\sqrt{\beta / 2} x] \exp [-i \sqrt{\beta / 2} x\rfloor, \quad \hat{\phi} \cong \beta^{-1 / 2} \hat{\phi}_{C}+\beta^{-1} \hat{\phi}_{L}
$$

where

$$
\hat{\phi}_{C}=\frac{\sqrt{i}}{k_{y}} \exp \left(k_{y} x\right), \quad \hat{\phi}_{L}=-i \exp \lfloor\sqrt{\beta / 2} x\rfloor \exp \lfloor-i \sqrt{\beta / 2} x\rfloor .
$$

Function $\hat{\eta}$, denoted as $\hat{\eta}_{L}$, is concentrated in the boundary layer $L$ of thickness $O\left(\beta^{-}\right.$ ${ }^{1 / 2}$ ). Function $\hat{\phi}$, on the other hand, consists of two terms. The first term in Eq. (25b) represents the $O\left(\beta^{-1 / 2}\right)$ core potential, which spreads much further from the wall, to a distance $O\left(k_{y}^{-1}\right)$. For $k_{y}=O(1)$, which is typical for reduction cells, the transverse size of the core is comparable to the wavelength of the disturbance. The second term in expression (25b) represents the $O\left(\beta^{-1}\right)$ boundary-layer correction to the core potential, which sets the normal component of current at the wall to zero.

Substituting further Eq. (25a) into Eq. (6a) gives the asymptotic expression for the real part of $\eta$ as follows:

$$
\eta \cong \exp [\sqrt{\beta / 2}(x \mp t)\rfloor \cos \left\lfloor\sqrt{\beta / 2}(x \mp t)-k_{y} y\right\rfloor .
$$

Note that as $\beta$ is high, the solution (27) satisfies the equation for a plane wave transverse to the wall, namely: 


$$
\frac{\partial^{2} \eta}{\partial t^{2}}=\frac{\partial^{2} \eta}{\partial x^{2}}
$$

Thus, the wall either 'radiates' or 'absorbs' a spatially decaying wave in the transverse direction. The source of 'radiation' is the Lorentz force on the right-hand side of the boundary condition (5b).

From Eq. (27) follows that the wave speed in the direction transverse to the wall equals to unity, i.e. to the phase speed of gravity waves. The longitudinal wave speed is equal to $k_{y}^{-1}(\beta / 2)^{1 / 2}=\left(\beta_{\lambda} / 2\right)^{1 / 2}$, which is much higher for $\beta_{\lambda}>>1$.

As the terms $O(1)$ in the expressions for $\omega$ and $k_{x}$ are purely imaginary, they affect the increment in the exponential term in expression (27) only, which becomes equal to $\sqrt{\beta / 2}-k_{y} / 2$. Thus the effect of the finite wavelength is to reduce the growth rate and to increase the distance from the wall affected by the disturbance.

\subsection{Mechanism sustaining the unstable wall mode}

Suppose that there is a disturbance of the interface, which is initially independent of the $x$-co-ordinate, as shown in Fig. 6 . Then the electric current is initially in the $\pm y$-direction, flowing from crests to troughs of the interface elevation. This current interacts with the magnetic field and the resulting Lorentz force pushes the fluid away from the wall on the right side of the crests. The fluid flows though the core, and returns to the wall at the left side of the crests. The resulting deficit of fluid on the right side leads to the depression of the interface, while the excess fluid on the left side creates a rise of the interface. As a result, the disturbance (i) starts growing in amplitude, (ii) propagates in the positive $y$-direction. 

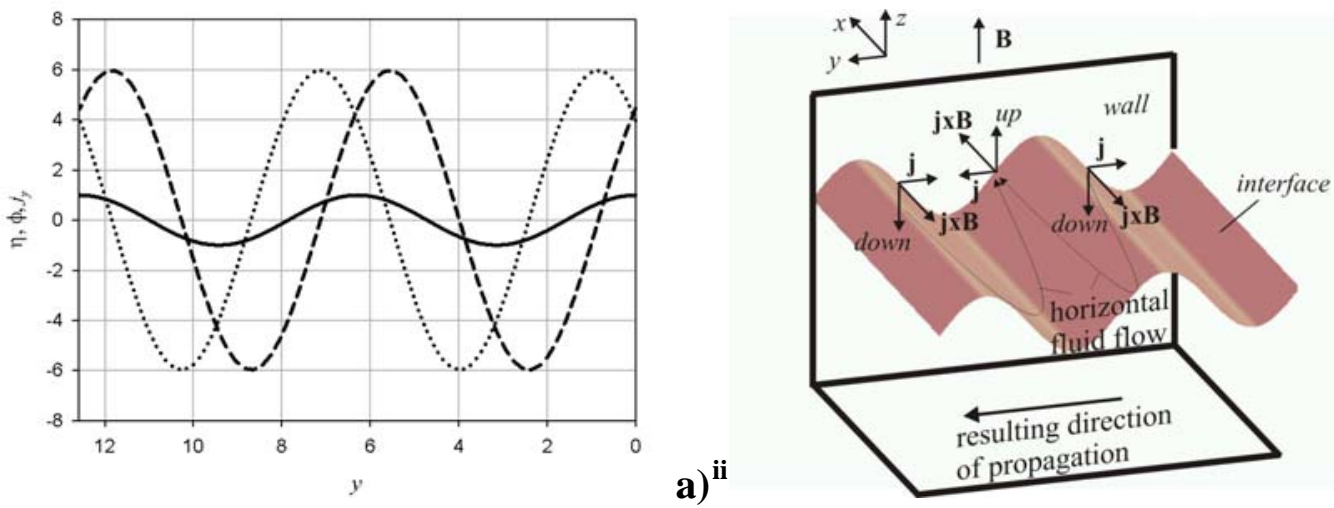

b)

FIG. 6. (a) Functions $\eta$ (solid line), $\phi$ (broken line), and $j_{y}$ (dotted line) at $x=0, t=0$ for $\beta=100$, and $k_{y}=1$ and (b) schematic diagram of the mechanism of instability

The source of the disturbance is the Lorentz force at the boundary, which results from the interaction between the core current with the background field. This current flows owing to the differences in the core potential, which is a global function. As the interface disturbance $\eta$ vanishes outside the boundary layer, the core current is purely horizontal. It drives the fluid globally from one part of the boundary to the other. Thus the core potential, shifted in phase to $\eta$, synchronises the propagation of the interface disturbance generated locally at various parts of the boundary.

Most of the results in subsections 3.2 and 3.3 have been obtained in [13] and [14]. They have been reproduced here as part of the unified theory of the phenomenon.

\section{Circular domain}

Another exact solution for travelling waves, which demonstrates important features of the modes, now in a closed domain, may be obtained for a circular geometry. Assuming

$$
\eta=\hat{\eta}(r) \exp (i n \theta-i \omega t), \quad \phi=\hat{\phi}(r) \exp (i n \theta-i \omega t),
$$

where 

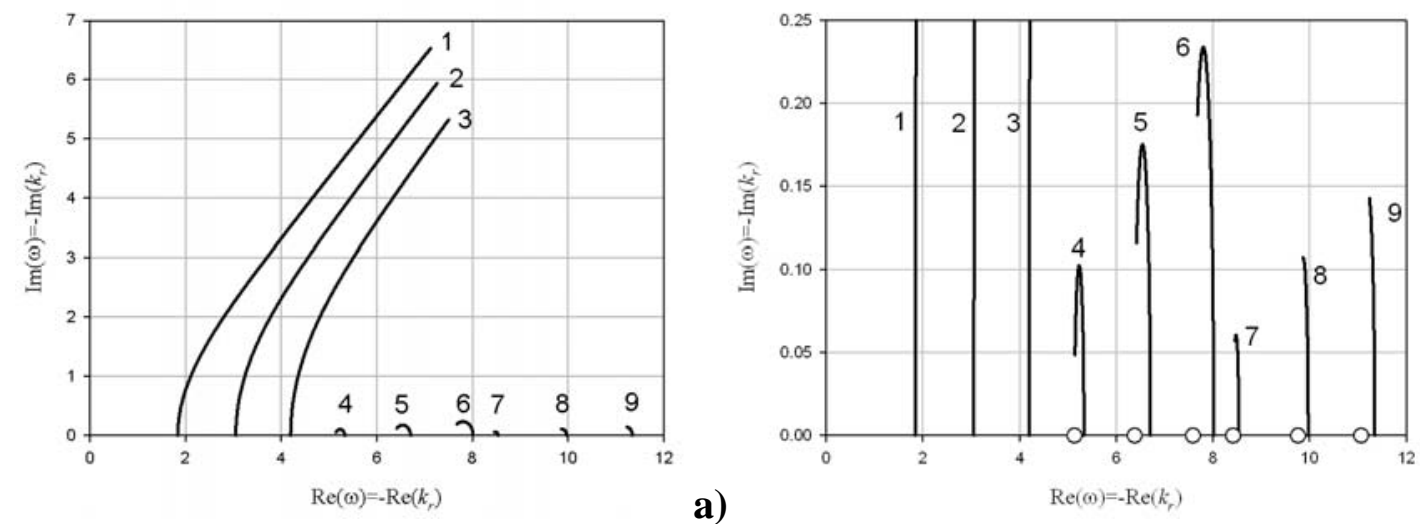

b)

FIG. 7. Variation of the first nine roots of the dispersion relation for a circular domain (a) and zoom into the Sele modes 4-9 (b). Modes number 1, 2, and 3 correspond to $n=1$ and $l=1,2,3$; modes number 4, 5, and 6 correspond to $n=2$ and $l=1,2,3$; modes number 7, 8, and 9 correspond to $n=3$ and $l=1,2,3$, respectively. The values of the roots on the real axis correspond to $\beta=0$, while the endpoints are for $\beta=100$. The limit values of the roots for modes 4-9 as $\beta \rightarrow \infty$ are shown with circles.

$$
k_{r}^{2}=\omega^{2}, \quad \hat{\eta}(r)=C J_{n}\left(k_{r} r\right), \quad \hat{\phi}(r)=k_{r}^{-2}\left\{\hat{\eta}(r)-n^{-1} \hat{\eta}^{\prime}(1) r^{n}\right\},
$$

$J_{n}(\bullet)$ is the Bessel function of the first kind [20], $n>0$ is an integer, $(r, \theta, z)$ are cylindrical co-ordinates, and ' $=d / d r$. The characteristic length $L^{*}$ is the radius of the circle. As the geometry is closed, the tangential, azimuthal wavenumber is now quantised and is equal to $2 n \pi$. The constant $C=1 / J_{n}\left(k_{r} r\right)$ has been selected in such a way that $\hat{\eta}(1)=1$. Parameter $k_{r}$ satisfies the following dispersion relation:

$$
i k_{r}^{2}-i n k_{r} J_{n}\left(k_{r}\right) / J_{n+1}\left(k_{r}\right)=-\beta \text {. }
$$

Note that the dispersion relation is similar to that for the half-plane. Indeed, introducing notation $f\left(k_{r}\right)=-i J_{n}\left(k_{r}\right) / J_{n+1}\left(k_{r}\right)$, gives:

$$
i k_{r}^{2}+n k_{r} f\left(k_{r}\right)=-\beta \text {. }
$$

For $f \equiv 1, k_{r}=k_{x}$, and $n=k_{y}$, Eq. (32) reduces to Eq. (21) exactly. Thus function $f$ expresses the effect of the geometry of the domain. 
Now, for each value of $n$ there is an infinite number of roots $k_{r}^{(n, l)}, l=1,2, \ldots$ of the dispersion relation (31). Both real and imaginary parts of the roots are either positive, or equal in modulus but negative, as the dispersion relation is invariant with respect to the transformation $k_{r} \mapsto-k_{r}$. In both cases the roots lead to the same solution. For compatibility with the half-plane problem we will be concerned with the latter roots, i.e. $\operatorname{Re}\left(k_{r}\right)=-\operatorname{Re}(\omega)<$ $0, \operatorname{Im}\left(k_{r}\right)=-\operatorname{Im}(\omega)<0$ (Fig. 6)

As $\beta$ increases, for each value of $n$ the roots with $l=1$ behave differently from those with $l \geq 2$ (Fig. 7). For $l=1$ both real and imaginary parts of $k_{r}^{(n, l)}$ grow in modulus. For $l \geq 2$, the real parts of the roots monotonically decrease in modulus, while the imaginary ones first grow, and then decrease. Thus there are two distinct groups of modes which are discussed below.

\subsection{Wall modes}

Consider the modes with $l=1$ first. For a fixed $n$ and for $\beta>>1$, Eq. (31) implies that $\left|k_{r}\right|>>1$ and that [20]

$$
J_{n}\left(k_{r}\right) \cong \frac{\exp \left(i k_{r}-\frac{1}{2} n \pi-\frac{1}{4} \pi\right)}{\sqrt{2 \pi k_{r}}}+\ldots, \quad J_{n}\left(k_{r}\right) / J_{n+1}\left(k_{r}\right) \cong i
$$

while the dispersion relation reduces to that for the half-plane exactly, i.e.

$$
i k_{r}^{2}+n k_{r}=-\beta,
$$

The asymptotic expressions for $k_{r}, \omega, \hat{\eta}$ and $\hat{\phi}$ obtained from the exact solution for the unstable modes are: 


$$
\begin{gathered}
k_{r}=-\omega \cong-\sqrt{\beta i}=-\sqrt{\frac{\beta}{2}}(1+i), \\
\hat{\eta} \cong \hat{\eta}_{L}=\exp [\xi \sqrt{-i}], \\
\hat{\phi} \cong \beta^{-1 / 2} \hat{\phi}_{C}+\beta^{-1} \hat{\phi}_{L},
\end{gathered}
$$

where

$$
\xi=\beta^{1 / 2}(r-1) \leq 0, \quad \hat{\phi}_{C}=\frac{r^{n} \sqrt{i}}{n}, \quad \hat{\phi}_{L}=-i \exp |\xi \sqrt{-i}| .
$$

The asymptotics is identical in structure to that for the half-plane.

The isolines of $\eta$ and $\phi$, based on the exact solution, are shown in Fig. 8a,b for the unstable wall mode with $n=1, l=1$ and $\beta=100$. This is the mode with the highest growth rate and the longest possible azimuthal wavelength of $2 \pi$, the perimeter of the domain. The whole pattern rotates counter-clockwise, as shown in the figure.

Function $\eta$ exhibits characteristic tails, similar to the pattern for the half-plane shown in Fig. 5c. The real part of the core potential,

$$
\phi_{C} \cong \beta^{-1 / 2} \exp [\sqrt{\beta / 2} t]\{x \sin [\sqrt{\beta / 2} t+\pi / 4\rfloor+y \sin [\sqrt{\beta / 2} t-\pi / 4]\},
$$




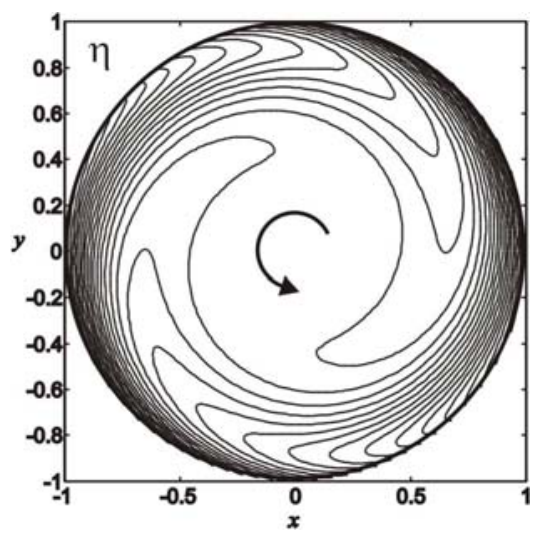

(a)

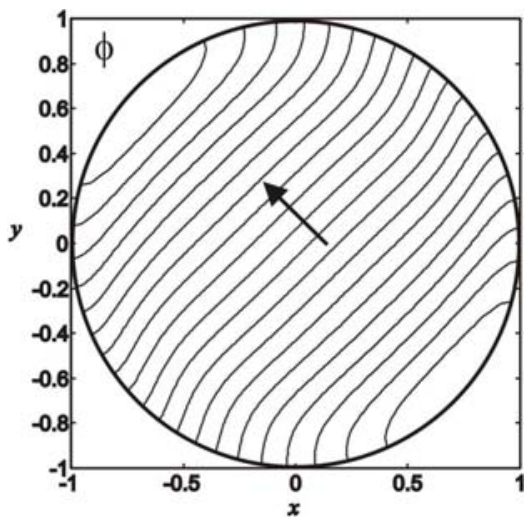

(b)
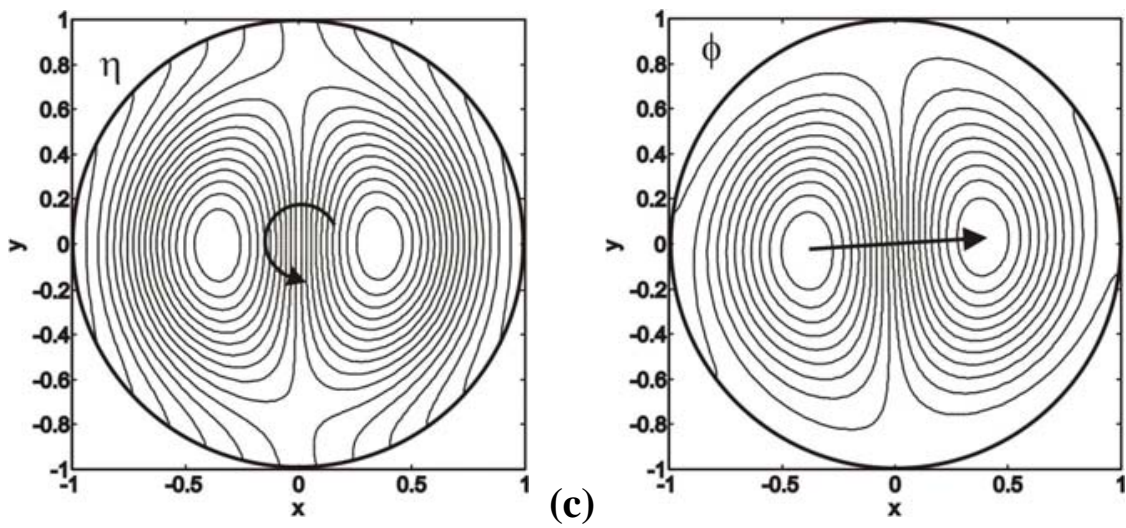

(d)

FIG. 8. Circular domain: isolines of $\eta$ and $\phi$ for the wall mode with $n=1, l=1$ (a), (b) and for the centre mode with $n=2, l=1$ (c), (d) for $\beta=100, t=0$. The pattern rotates counter-clockwise. The arrows across the isolines of $\phi$ show schematically the direction of the core current.

is a global function, and is linear in both $x$ and $y$. The electric current in the core being independent of either $x$ or $y$ flows in straight lines transverse to the isolines of the core potential (Fig. 8b). Interaction of the current with the background field leads to the horizontal flow of aluminium from the trough to the crest of $\eta$ through the core, transverse to the isolines of $\phi$.

Figure 9 shows the results for a square domain obtained numerically with a finite difference method (second-order accurate in both space and time, based on central differences throughout). Calculations were performed for sufficiently long time so that the most unstable mode takes over all the other modes with lower growth rate. It is clear that the results shown in Fig. 8a,b for a circle and in Fig. 9 for a square are essentially the same topologically. 


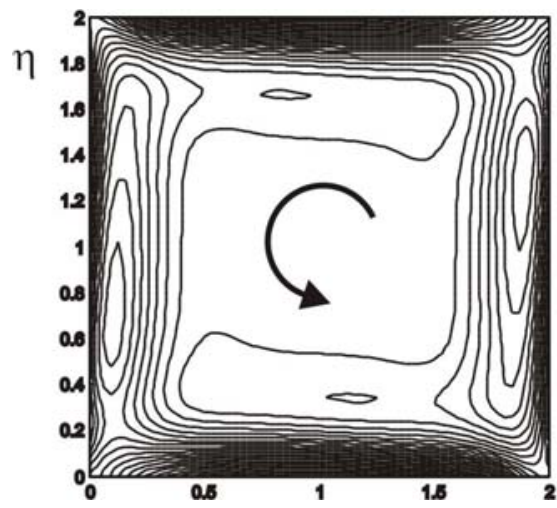

a)

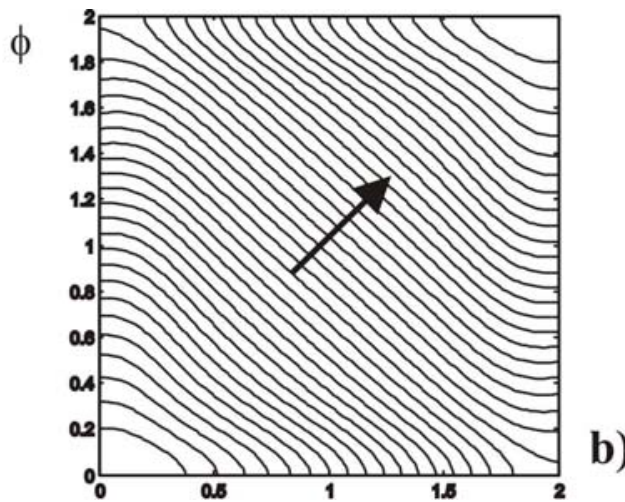

FIG. 9. Instability in a square domain: isolines of $\eta$ (a) and $\phi$ (b) for $\beta=100$ at $t=8.2$. The pattern rotates counter-clockwise. The arrows across the isolines of $\phi$ show schematically the direction of the core current.

\subsection{Centre, or Sele modes}

The roots of the dispersion relation, which define modes with $l \geq 2$, as well as the resulting solution, behave drastically different from those with $l=1$. They start at the real axis for $\beta=0$, and return to this axis as $\beta \rightarrow \infty$ (Fig. 7). In fact, for high values of $\beta$ they are characterised by the following asymptotics:

$$
k_{r} \cong k_{0}+\beta^{-1} k_{1}+\ldots
$$

where both $k_{0}$ and $k_{1}$ are $O(1)$ and $k_{0}$ is real. For these modes both functions $\hat{\eta}$ and $\hat{\phi}$ are $O(1)$ in the core, which means that the magnitude of potential is determined by Eq. (1b). Thus most of the disturbance current completes its path in the core between maxima and minima of $\phi$ (Fig. 8d), which are located inside the domain, rather than at the boundary. As both $\hat{\eta}$ and $\hat{\phi}$ are $O(1)$, then from Eqs. $(30 \mathrm{c}, \mathrm{b})$ follows that to the leading order

$$
\hat{\phi}=0 \quad \text { at } r=1,
$$

which implies vanishing tangential component of current at the boundary. 
From Eq. (31) follows that in the limit $\beta \rightarrow \infty$ for $\left|k_{r}\right|=O(1)$ the dispersion relation becomes

$$
J_{n+1}\left(k_{0}\right)=0,
$$

The roots of this equation lie close to those of Eq. (31) (see Fig. 7) and "attract" these roots.

From Eqs. (30) and (35) follows that in the limit $\beta \rightarrow \infty$ the boundary condition for the $O(1) \hat{\eta}$ is:

$$
\hat{\eta}^{\prime}(1)=n \hat{\eta}(1)
$$

Thus, despite the fact that $k_{0}$ is real, these are clearly not the gravity waves. Neither these are the modes for a strictly perfectly conducting wall as would be implied by Eq. (35). In fact, these modes may be qualified as the rotating interface first studied by Sele [3]. Indeed, these are the waves with non-zero elevation in the entire domain, which rotate with a single frequency. Although such waves are unstable, they are by far less dangerous than the wall modes as their growth rate, $O\left(\beta^{-1}\right)$, will be limited by dissipation.

The centre modes are almost fully analogous to those from the continuous spectrum for the half-plane with the notable exception that they are weakly unstable. We will clarify the reason for this in Sec. 6 but before that we will consider instability in an infinite channel as it brings in new features of instability. 


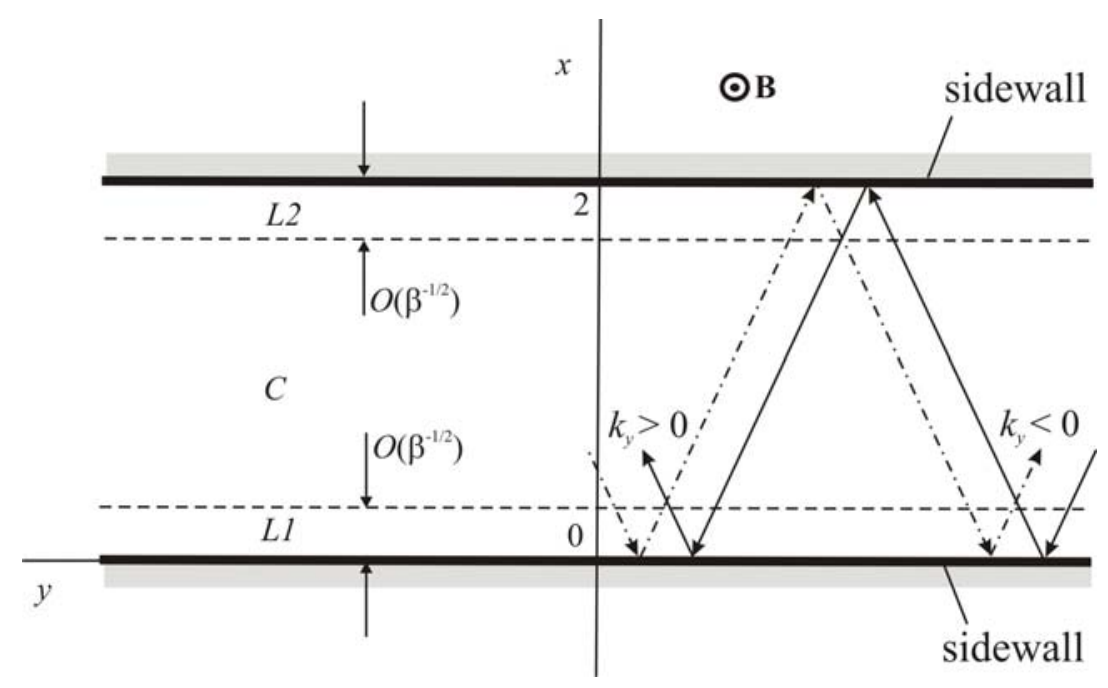

FIG. 10. Schematic diagram of the channel problem. On the left are flow subregions for the wall modes for $\beta \gg 1$ : boundary layers $L 1, L 2$, and core $C$; on the right is schematic diagram of reflection of plane waves from the sidewalls

\section{Infinite channel}

Schematic diagram of an infinite channel is shown in Fig. 10. What is new here with respect to the geometries considered before is that there are two separated boundaries, each of which generates unstable waves.

The problem for the travelling waves is defined by Eqs. (7) subject to the boundary conditions

$$
\frac{d \hat{\phi}}{d x}=0, \quad \frac{d \hat{\eta}}{d x}=-i \beta k_{y} \hat{\phi} \quad \text { at } \quad x=0 \text { and at } x=2 \text {, }
$$

and the normalization condition

$$
\hat{\eta}(2)=1
$$

The characteristic length $L^{*}$ is half the channel width.

An exact solution to the problem in the form of travelling waves [10] is represented here in the following form:

$$
\hat{\eta}=C_{3} e^{i k_{x} x}+C_{4} e^{-i k_{x} x}
$$



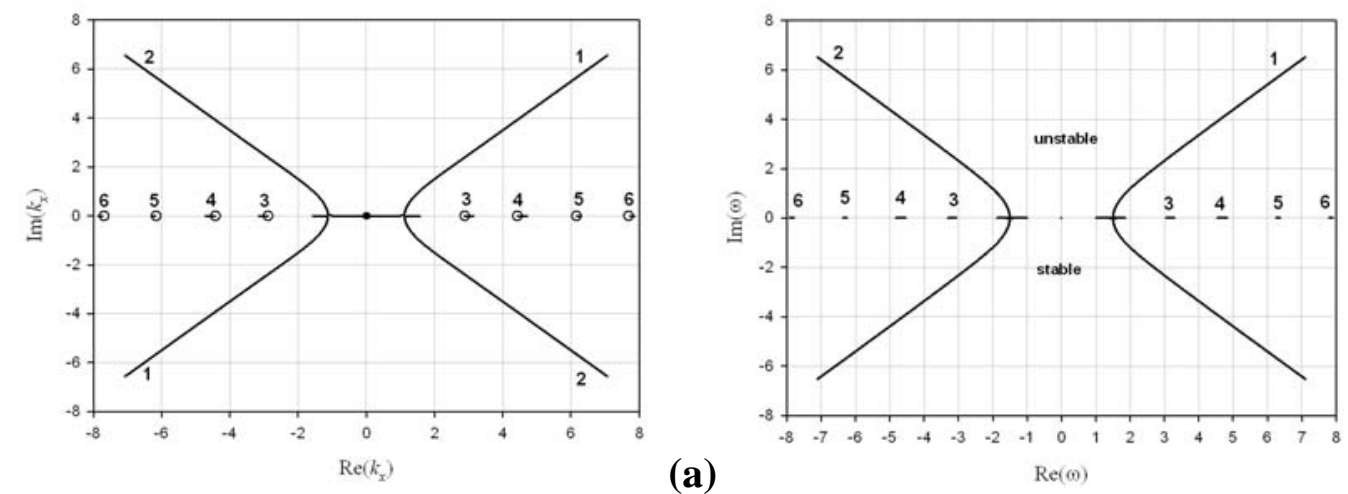

(b)

FIG. 11 Instability in an infinite channel: variation of the first four roots of the dispersion relation (42) (a) and corresponding values of $\omega$ (b) with $\beta$ for $k_{y}=1$ and for $\operatorname{Re}\left(k_{x}\right)<0$. The values of the roots on the real axis correspond to $\beta=0$, while the endpoints are for $\beta=100$. The values of $k_{x}$ as $\beta \rightarrow \infty$ are shown with circles.

$$
\hat{\phi}=\frac{1}{\omega^{2}}\left\{C_{3} e^{i k_{x} x}+C_{4} e^{-i k_{x} x}\right\}+C_{5} e^{k_{y} x}+C_{6} e^{-k_{y} x}
$$

where $k_{x}^{2}=\omega^{2}-k_{y}^{2}$, and $C_{3}-C_{6}$ are constants.

The boundary conditions (38a-d) and the normalization condition (39) yield the spatial exponents $k_{x}$ as the roots of the dispersion relation

$$
16 \beta^{2}\left\{2 p q(\cos p \cosh q-1)+\left(p^{2}-q^{2}\right) \sinh q \sin p\right\}+p^{2}\left(p^{2}+q^{2}\right)^{2} \sinh q \sin p=0,
$$

where $p=2 k_{x}, q=2 k_{y}$, and the values for $C_{3}-C_{6}$, whose lengthy expressions are not presented here. The dispersion relation is invariant with respect to transformation $k_{x} \mapsto-k_{x}$.

Variation of the spatial exponents and the corresponding values of $\omega$ are shown in Fig. $11 \mathrm{a}$ and $11 \mathrm{~b}$, respectively, for $k_{y}=1$ and for varying $\beta$. Similar to the flow in a circular domain, there are two types of roots. First of all, only roots 1 and 2 exhibit strong growth in modulus for increasing $\beta$, and thus represent the unstable modes. There is also an infinite number of roots, which remain real for all values of $\beta$. The first four roots belonging to this group are shown in Fig. 11 (lines 3-6).

\subsection{Wall modes}


Consider roots 1 and 2 first for $\operatorname{Re}\left(k_{x}\right)<0$. In contrast to the flow in a circular domain, which is unstable for any non-zero value of $\beta$, there is a threshold of instability for the waves in a channel. For $k_{y}=1$ roots 1 and 2 remain real below $\beta_{c r}=1.6$. At $\beta=\beta_{c r}$ an internal resonance occurs as a result of which the imaginary parts of $k_{x}$ and $\omega$ appear and the flow becomes unstable.

As the two boundaries, located at $x=0$ and $x=2$ are not connected, two separate boundary layers are formed for $\beta>>1$. They are denoted by $L 1$ and $L 2$, respectively (Fig. 10).

We will be looking for the asymptotic solution as follows:

$$
\begin{gathered}
\hat{\eta} \cong \hat{\eta}_{L 1}+\hat{\eta}_{L 2}, \\
\hat{\phi} \cong \beta^{-1 / 2} \hat{\phi}_{C}+\beta^{-1}\left(\hat{\phi}_{L 1}+\hat{\phi}_{L 2}\right),
\end{gathered}
$$

where subscripts denote the region of validity of the respective terms. The second and the third terms in Eq. (44) are boundary-layer corrections to the core potential.

In the boundary layers and the core the governing equations are:

$$
\begin{gathered}
\frac{\partial^{2} \hat{\eta}_{L 1}}{\partial \xi^{2}}+\kappa^{2} \hat{\eta}_{L 1}=0, \quad \frac{\partial^{2} \hat{\phi}_{L 1}}{\partial \xi^{2}}=-\hat{\eta}_{L 1} \quad \text { in layer } L 1, \\
\frac{\partial^{2} \hat{\eta}_{L 2}}{\partial \zeta^{2}}+\kappa^{2} \hat{\eta}_{L 2}=0, \quad \frac{\partial^{2} \hat{\phi}_{L 2}}{\partial \zeta^{2}}=-\hat{\eta}_{L 2} \quad \text { in layer } L 2, \\
\frac{d^{2} \hat{\phi}_{C}}{d x^{2}}-k_{y}^{2} \hat{\phi}_{C}=0 \quad \text { in the core, }
\end{gathered}
$$

where $\kappa=\beta^{-1 / 2} k_{x}=O(1)$, and $\xi=-\beta^{1 / 2} x \leq 0, \zeta=\beta^{1 / 2}(x-2) \leq 0$ are stretched co-ordinates in layers $L 1$ and $L 2$, respectively. 
Integrating Eqs. (45), and taking into account the normalization condition (39) yields the general solution as follows:

$$
\begin{gathered}
\hat{\eta}_{L 1}=C_{7} \exp (i \kappa \xi), \quad \hat{\phi}_{L 1}=\kappa^{-2} \hat{\eta}_{L 1}, \\
\hat{\eta}_{L 2}=\exp (i \kappa \zeta), \quad \hat{\phi}_{L 2}=\kappa^{-2} \hat{\eta}_{L 2}, \\
\hat{\phi}_{C}=A_{1} \exp \left\{-k_{y} x\right\}+A_{2} \exp \left\{k_{y}(x-2)\right\},
\end{gathered}
$$

where $C_{7}, A_{1}$, and $A_{2}$ are arbitrary constants. Constant $C_{7}$ determines the value of $\hat{\eta}$ at $x=0$. These constants, as well as the value of $\kappa$, are determined from the four boundary conditions (38a-d). In particular, it follows that $\kappa^{2}= \pm i$.

In order for the boundary-layer terms to decay away from respective sidewalls, we require that $\operatorname{Im}(\kappa)<0$. This leads to two possible values for $\kappa$, namely:

$$
\kappa=2^{-1 / 2}( \pm 1-i)= \pm \sqrt{\mp i}
$$

which correspond to roots 1 and 2 of the dispersion relation, respectively. Thus we arrive at two different solutions for $\hat{\eta}$ and $\hat{\phi}$.

In the first case, i.e. for $\kappa=2^{-1 / 2}(1-i)$, the result is as follows:

$$
C_{7}=-\exp \left(2 k_{y}\right), \quad A_{1}=-\kappa / k_{y} \exp \left(2 k_{y}\right), \quad A_{2}=0,
$$

while in the second case, i.e. for $\kappa=-2^{-1 / 2}(1+i)$, one gets

$$
C_{7}=-\exp \left(-2 k_{y}\right), \quad A_{1}=0, \quad A_{2}=-\kappa / k_{y} .
$$

Substituting expressions (46)-(48) into Eqs. (43) and (44), and using the values of constants from Eqs. (50) or (51), yields: 

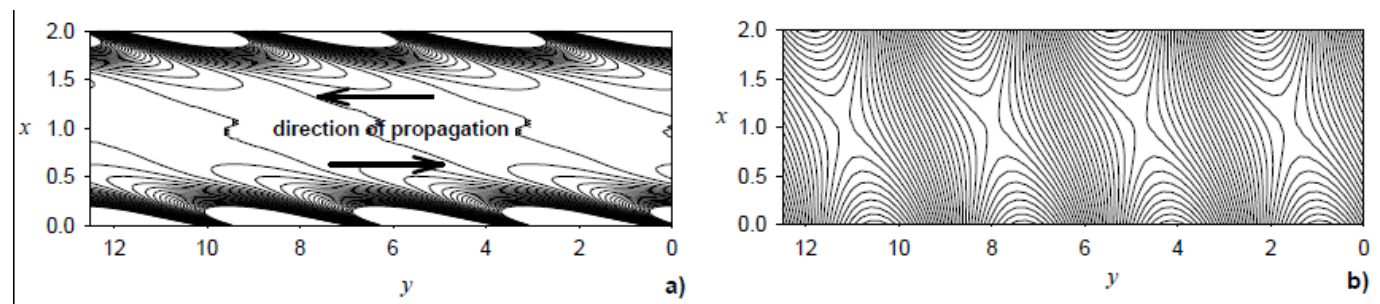

FIG 13. Infinite channel. Contour lines of $\eta$ (a) and $\phi$ (b) for combined asymptotic solution (55) for $k_{y}=1, \beta=100, t=0$. The disturbance travels in the $+y$-direction at the wall $x=2$ and in the $-y$ direction at the wall $x=0$. .ii $^{\text {ii }}$

$$
\begin{gathered}
\hat{\eta} \cong-\exp \left\lfloor \pm 2 k_{y}+i \kappa \xi\right]+\exp (i \kappa \zeta), \\
\hat{\phi} \cong-\beta^{-1 / 2} \frac{\kappa}{k_{y}} \exp \left[ \pm k_{y}(2-x)\right] \pm \beta^{-1} i\left\{-\exp \left[ \pm 2 k_{y}+i \kappa \xi\right]+\exp (i \kappa \zeta)\right\},
\end{gathered}
$$

where the upper and lower signs are taken for the first or the second solution as defined by Eq. (49).

As $\omega^{2} \cong \beta \kappa^{2}$, for each value of $\kappa$ there are two possible values of $\omega$. The one with $\operatorname{Im}(\omega)>0$ gives a temporally growing wave, while that with $\operatorname{Im}(\omega)<0$ gives a decaying wave. Thus for the unstable modes,

$$
\omega=-\beta \kappa=(\beta / 2)^{1 / 2}(\mp 1+i) .
$$

The sign convention is as in Eq. (49). The unstable waves defined by solutions 1 and 2 travel in the $-y$ and $+y$-directions, respectively. The result (54) has been obtained in [13] in the long-wave approximation, $k_{y} \rightarrow 0$ and independently in [14] for $\beta \rightarrow \infty$.

The isolines of $\eta$ and $\phi$ for solution 2 are shown in Fig. 12 for $\beta=100$ and for $k_{y}=1$, and $t=0$. The solution is clearly asymmetric about the midplane of the channel $x=1$. This asymmetry is fundamental, as symmetric or anti-symmetric unstable boundary modes cannot propagate along the channel strictly in the $+y$ or $-y$-direction. For such waves the amplification at one wall is accompanied by equal in magnitude suppression at the opposite one. 
However, counter-propagating, unstable, anti-symmetric waves in $(x-1)$ are not only admissible, but are directly relevant to the flow in a rectangular domain. Such a wave may be represented by a linear combination of the two solutions obtained in the above. This gives:

$$
\left\{\begin{array}{l}
\eta \\
\phi
\end{array}\right\}=\frac{\exp \left(-k_{y}\right)}{2 \cosh k_{y}}\left\{\begin{array}{l}
\eta_{1} \\
\phi_{1}
\end{array}\right\}+\frac{\exp \left(k_{y}\right)}{2 \cosh k_{y}}\left\{\begin{array}{l}
\eta_{2} \\
\phi_{2}
\end{array}\right\}
$$

where subscripts 1 and 2 correspond to solutions 1 and 2, respectively. The isolines of functions $\eta$ and $\phi$ for such a wave at $t=0$ are shown in Fig. 13.

\subsection{Centre modes}

Besides two complex roots of the dispersion relation (42), there is an infinite number of roots, which remain real for all values of $\beta$. These roots correspond to the Sele modes. The first four roots of this type are 3-6 (Fig. 11). Corresponding solutions are characterised by both $\hat{\eta}$ and $\hat{\phi}$ being $O(1)$ quantities in the interior of the domain. As $\beta \rightarrow \infty$, function $\hat{\phi}$ satisfies the boundary conditions $\hat{\phi}=0$ and $d \hat{\phi} / d x=0$ at both boundaries $x=0$ and $x=2$. This gives the dispersion relation as follows:

$$
2 p q(\cos p \cosh q-1)+\left(p^{2}-q^{2}\right) \sinh q \sin p=0,
$$

which may also be obtained from Eq. (42) in the limit $\beta \rightarrow \infty$ for $\left|k_{x}\right|=O(1)$.

The roots of Eq. (56), shown in Fig. 11a with circles, attract those of Eq. (42). Therefore, there is a full analogy between the higher roots for a channel, and those for a circle, except perhaps one: in the circular domain the corresponding modes are weakly unstable, while for a channel they are stable. We will return to the question as to why this is the case in Sec. 6. 


\section{Reflection mechanism}

Here a unified view on the nature of stable or weakly unstable modes for various geometries is outlined. It allows to understand their properties in terms of wave reflection from the boundary.

\subsection{Circular domain}

The direct connection of the growth rate $|\operatorname{Im} \omega|=\left|\operatorname{Im} k_{r}\right|$ of the centre modes in a closed geometry and the reflection coefficient can be extracted by considering short waves in the circle (see Sec. 4) corresponding to $n \sim \sqrt{\beta}>>1, \quad l>1$ For these modes, $\left|\operatorname{Re} k_{r}\right| \sim \sqrt{\beta}>>\left|\operatorname{Im} k_{r}\right|$ (i.e. nearly periodic short waves hence 'geometric optics' is relevant), and the following relationship follows from the relevant decomposition of the exact solution (25a) (see [12]):

$$
\mu=\exp \left(4\left|\operatorname{Im} k_{r}\right|\right)=\frac{\beta_{\lambda}{ }^{2}+\left(1+\beta_{\lambda} n / \operatorname{Re} k_{r}\right)^{2}}{\beta_{\lambda}{ }^{2}+\left(1-\beta_{\lambda} n / \operatorname{Re} k_{r}\right)^{2}}
$$

Formula (57) establishes a direct connection of the weak instability of the higher centre modes with the reflection mechanism. Note that $\beta_{\lambda}=\beta /\left(\operatorname{Re} k_{r}\right)^{2}=O(1)$ which implies $\mu-1=O(1)$ even for high $\beta$ and makes the rotating modes with higher $n$ more unstable than those with lower $n$. This is also in accordance with the behaviour of the higher roots of the exact dispersion relation for the circle (see Fig. 7).

In conclusion we note that from a comparison of Eq. (57) with Eq. (13) it follows that the equivalent angle of incidence for the higher centre modes is $\tan \vartheta=n / \operatorname{Re} k_{r}=O(1)$. 


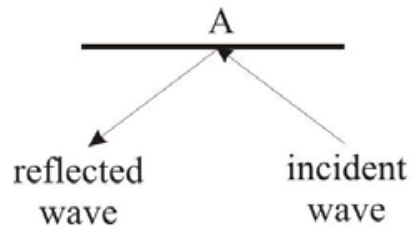

a)

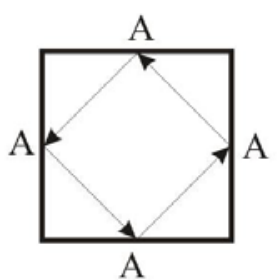

d)

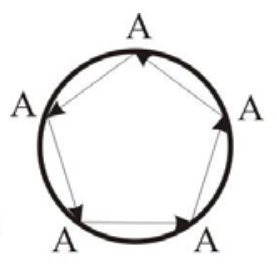

b)

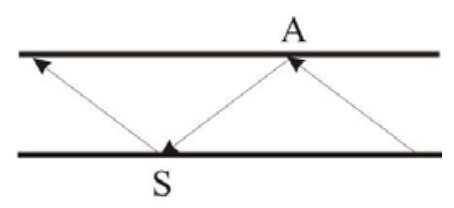

c)

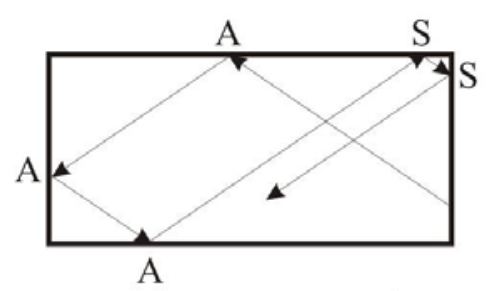

e)

FIG. 14. Schematic diagram of reflection of short waves in various geometries: half-plane (a), circle (b), channel (c), square (d), and rectangle (e). The wave is amplified at points A, and suppressed at points $\mathrm{S}$.

\subsection{Applications of the reflection theory}

As has been mentioned at the end of Sec. 5, the origin of the centre modes for a circle and a channel is similar. The qualitative difference between the two geometries is that these modes are stable for a channel and weakly unstable for a circle. This difference may well be explained by the reflection mechanism.

First of all, it has been established in Sec. 3.1 that for real values of $k_{x}$, a wave reflected from a plane wall, as shown in Fig. 14a, would be amplified. If the wave approaches the wall in the reverse direction it would be suppressed.

For a circle, any wave reflected from the boundary in the counter-clockwise direction is amplified (Fig. 14b). Then a reflected wave travels towards the other point of the same boundary, and is amplified again. As a result of multiple reflections, the amplitude of the wave grows, and all the modes are unstable [12]. 
For a channel (Fig. 14c), a wave amplified at one of the walls, reflects from a different wall. At that wall the local angle of reflection changes to the opposite, and the wave is suppressed. Owing to symmetry, the total coefficient of reflection from the two walls is equal to unity, and the wave remains stable.

For a square (Fig. 14d) any wave propagating counter-clockwise is amplified as the 'rays' form a closed trajectory. This is why the equilibrium is unstable for any non-zero value of $\beta$, similar to a circle.

Concerning a rectangle (Fig. 14e), only some of the modes would grow in amplitude with time, namely those able to form a closed trajectory. The one shown schematically in Fig. 14e would not be. This would ultimately depend on the aspect ratio of the rectangle. As the increasing MHD interaction changes the angle of incidence, the trajectory for some of the short waves becomes closed, and the flow becomes unstable.

Therefore, the interpretation of instability in terms of reflection from the boundary is very useful for understanding several important features. It helps to explain (i) why some modes are stable, while the others are not, (ii) why equilibrium in certain geometries is unstable for any non-zero value of $\beta$, while in the others there is a threshold of instability, (iii) why equilibrium in an unbounded domain is stable, etc. As other simplified models do not take the boundary into account, they fail to predict many of these features.

\section{Discussion and conclusions}

We have presented a unified view of interfacial MHD instability in a model of aluminium reduction cells with a uniform magnetic field. For any horizontal cross-section of the cell there are two distinct groups of travelling waves, or modes. The wall modes resulting from the interaction of the mechanical and the electromagnetic quantities at the electrically 
insulating boundary form the first group. These waves are most unstable with the growth rate being $O\left(\beta^{1 / 2}\right)$ as $\beta \rightarrow \infty$. The key element for the development of this type of instability is the Lorentz force which carries the fluid from the right side of the crests at the sidewall to the left side resulting in anti-clockwise propagation of the wave. As the fluid flows through the core, it is accelerated by an unopposed Lorentz force and this leads to the amplification of the wave and thus to the instability.

The second group of travelling waves results from coupling of the mechanical and the electromagnetic quantities inside the whole domain. For a finite value of $\beta$ these modes are either stable or weakly unstable, which can well be predicted by the reflection theory. In closed domains there are always weakly unstable modes of this type, which obey Sele's mechanism of instability. These Sele modes are characterised by the $O(1)$ disturbance of the interface and the electric potential in the whole domain, by vanishing tangential component of current at the sidewall, and by the growth rate being $O\left(\beta^{-1}\right)$ as $\beta \rightarrow \infty$. Thus they are ultimately damped by the magnetic field.

For both types of modes instability occurs only in the presence of an electrically insulating boundary. 
[1] Sele, T.: Instabilities of the metal surface in electrolytic aluminium reduction cells. Metallurgical Transactions B, 8B, 613-618 (1977) ${ }^{\text {iv }}$

[2] Gerbeau, J.-F., Le Bris, C., Lelievre, T.: Mathematical Methods for the Magnetohydrodynamics of Liquid Metals. Oxford Univ Press, New York (2006)

[3] Davidson, P.A.: Overcoming instabilities in aluminium reduction cells: a route to cheaper aluminium, Mater. Sci. Technol., 16, 475-479 (2000)

[4] Lukyanov, A., El, G., Molokov, S.: Stabilisation of the liquid metal - electrolyte systems. Int. Patent Application No. PCT/GB2003/00072 (2003)

[5] Urata, N.: Magnetics and metal pad instability. Light Metals, 581-591 (1985)

[6] Sneyd, A.D., Wang, A.: Interfacial instability due to MHD mode coupling in aluminium reduction cells. J. Fluid Mech., 236, 111-126 (1994)

[7] Bojarevics, V., Romerio, M.V.: Long wave instability of liquid metal-electrolyte interface in aluminium reduction cells: a generalisation of Sele's criterion. Eur. J. Mech. B/Fluids, 13, 33-56 (1994)

[8] Bojarevics, V.: Nonlinear waves with electromagnetic injection in aluminium electrolysis cells. In: Progr. in Astronautics and Aeronautics, 182, 833-848 (1998)

[9] Droste, Ch., Segatz, M., Vogelsang, D.: Improved 2-dimensional model for magnetohydrodynamic stability analysis in reduction cells. Light Metals, 419-428 (1998)

[10] Davidson, P.A., Lindsay, R.L.: Stability of interfacial waves in aluminium reduction cells. J. Fluid Mech., 362, 273-295 (1998)

[11] Zikanov, O., Thess, A., Davidson, P.A., Ziegler, D.P.: Nonlinear shallow water model of the interfacial instability in aluminium reduction cells. In: Proc. $3^{\text {rd }}$ Int. Symposium on Electromagnetic Processing of Materials, Nagoya, Japan, 2000, 109-114 (2000)

[12] Lukyanov, A., El, G., Molokov, S.: Instability of MHD-modified interfacial waves revisited. Physics Letters A, 290, 165-172 (2001)

[13] Morris, S.J.S., Davidson, P.A.: Hydromagnetic edge waves and instability in reduction cells, J. Fluid Mech., 493, 121-130 (2003)

[14] Molokov, S., El, G., Lukyanov, A.: On the nature of interfacial instability in aluminium reduction cells. Coventry University, Internal Report AM-01/2003, October 2003 
[15] Sun, S., Zikanov, O., Finlayson, B.A. Effect of background melt flow and interface distorsion on the stability of Hall-Herault cells. Magnetohydrodynamics, 41, 273287 (2005)

[16] Kohno, H., Molokov, S.: Finite element analysis of interfacial instability in aluminium reduction cells in a uniform, vertical magnetic field. Int J Engng Sci 45, 644-659 (2007)

[17] Pedcenko, A., Molokov, S., Thomas, P.J., Lukyanov, A., Priede, J.: Experimental study of interfacial instability in aluminium reduction cells. In: Proc. 7th PAMIR Conference on Fundamental and Applied MHD, Presquile de Giens, France, September 8-12, 2008, 923-927 (2008)

[18] Dupuis, M., Bojarevics, V., Freibergs, J.: Demonstration thermo-electric and MHD mathematical models of a $500 \mathrm{kA}$ Al electrolysis cell. In: Proc. $42^{\text {nd }}$ Conf. Metallurgists COM 2003, Vancouver, Canada, 1 - 18 (2003)

[19] Sneyd, A. D.: Interfacial instabilities in aluminium reduction cells. J Fluid Mech 236111-126 (1992)

[20] Abramowitz, M., Stegun, I.A.: Handbook of Mathematical Functions. Nat. Bureau of Standards. (1964) 